\title{
Digitalization as a driver for supporting PV deployment and cost reduction
}

\author{
Erika Saretta ${ }^{1, *}$, Pierluigi Bonomo ${ }^{1}$, Willy Maeder $^{2}$, Van Khai Nguyen $^{2}$, and Francesco Frontini ${ }^{1}$ (B) \\ ${ }^{1}$ SUPSI-DACD-ISAAC, 6850 Mendrisio, Switzerland \\ ${ }^{2}$ CADCAMATION, 1213 Onex, Switzerland
}

Received: 30 June 2021 / Received in final form: 9 December 2021 / Accepted: 13 December 2021

\begin{abstract}
Digitalization is providing advantages to all sectors around the world and it can be of relevance also for the photovoltaic (PV) sector. As an example, the current value chain of the European PV sector is often characterized by analogue and fragmented processes that should be overcame to support greater PV deployment. The adoption of a more open and collaborative digital-based approach characterized by datasharing among different stakeholders and more integrated information thread from the design till O\&M can provide direct benefits in optimizing the PV process, increasing performances, and reducing of costs. Therefore, a novel PV Information Management (PIM) approach has been drawn within the European H2020 project "SuperPV". In accordance with PIM objectives, a workflow for seamlessly transferring data along main PV workstages has been developed, as well as new digital features to specifically address collaborative approach in the PV sector such as: (i) advanced functionalities introduced in the existing BIMSolar ${ }^{\circledR}$ software for improving the simultaneous design, performance simulation and cost assessment of medium and large PV systems, (ii) a proofof-concept for aggregating all relevant information into a Digital Twin platform aimed at setting the ground for post-construction management and lifecycle assessment of the whole PV system.
\end{abstract}

Keywords: PV plant design / PV plant simulation / cost-assessment / PIM / digital twin

\section{Introduction}

The process of moving from analogue to digital technologies is affecting almost all industries around the world [1]. SolarPower Europe recognized digitalization as one of the key priorities for the PV sector. Indeed, digitalization is considered as a successful strategy for reducing PV asset management and operations and maintenance (O\&M) costs. Reported benefits are the better monitoring thanks to IoT that allows controlling the PV plant almost in real time and rapidly identification of issues, the automation of repetitive O\&M tasks, and the adoption of AI that allows anticipation of damages thus improving plant profitability $[2,3]$. Moreover, digitalization is adopted in the design stage of PV systems as reported in the full comprehensive review of [4], as well as to design the most cost-efficient BIPV solutions $[5,6]$.

Even though most solar stakeholders are fully aware of the potential benefits of digitalization, the full digitalization of the PV sector is not already achieved. If on one hand, "only $13 \%$ of solar experts claimed that lack of a digital mind-set was an obstacle to digitalization" [1], on

\footnotetext{
* e-mail: erika.saretta@supsi.ch
}

the other hand [7] highlighted that "the prevailing approach to digital innovation in energy is to create "point solutions," which apply technology to narrow uses without fundamentally changing how people work".

In this context, a real challenge can be identified in the development of holistic digital solutions that can be easily adopted by PV stakeholders and companies not only to improve a single stage of the PV process but to support the generation of an effective digital thread. The latter should be able to create a seamless information workflow that can be managed and passed through the various stages taking the opportunity to transform PV data into value.

In order to provide a valuable tool for PV and BIPV design, a BIM-based tool named BIMSolar ${ }^{(B)}$ was introduced in [8]. In that work, the concept of Building Information Modelling (BIM) was introduced to make PV and BIPV "interoperable" with the BIM approach and the whole building process but, at that stage, BIMSolar ${ }^{\circledR}$ doesn't address neither the typical PV process nor the topic of cost-reduction and PV data management along the whole PV process.

Therefore, a wider perspective named "PIM" approach has been defined within the European H2020 Project SuperPV [9] to embrace the main stages of the typical PV process for medium and large-scale PV systems and reduce 


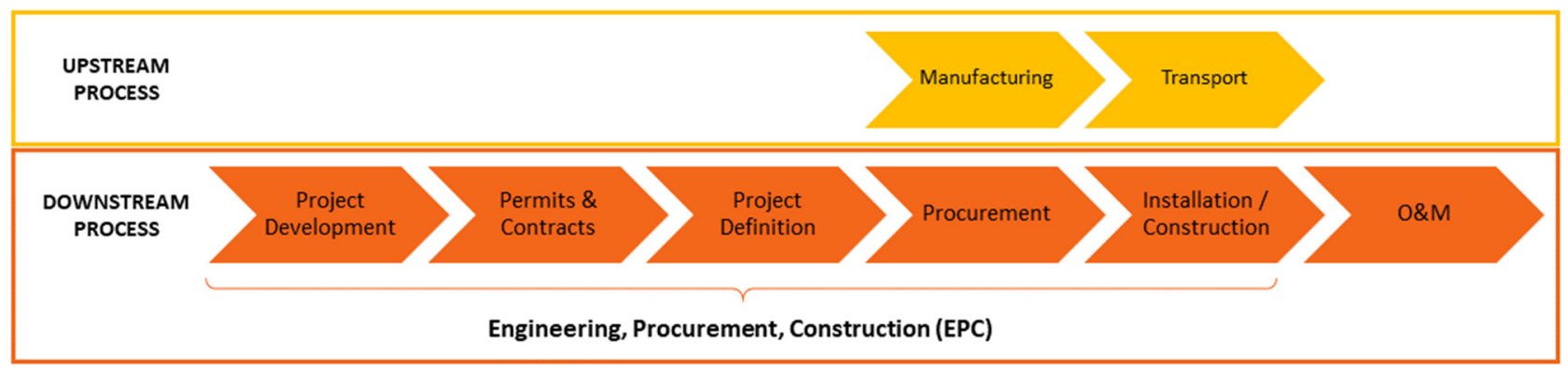

Fig. 1. Main stages of the current reference PV process.

the costs. Consequently, a new prototype collaborative platform for design, simulation and operation has been developed to increase the interoperability of the PV process and to support the cost reduction of PV systems.

In this paper, an overview on the PIM approach is firstly provided in Section 2. The implementation of this approach is then described through the introduction of the prototype collaborative platform for design, simulation and operation in Section 3, together with new features and functionalities for supporting cost-reduction. In Section 4, results about preliminary validation on a test case for assessing the design and simulation features and potential cost-reductions are presented, and in Section 5 some conclusions are drawn.

\section{Methodology: the "PIM" approach for PV cost reduction}

Digitalization can refer to a wide range of topics. Therefore, in the framework of the SuperPV project a fundamental preliminary action has been represented by the definition of what digitalization means for the PV process. In particular, to address digitalization in the $\mathrm{PV}$ value chain, the definition of the map of the current PV process and the identification of current main bottlenecks and needs for improvements have been carried out. Secondly, the needs and stakeholders' requirements have been used as a basis to define a digital approach named PIM that consists of a PV information management strategy to address efficiently relevant key-stage of the PV process towards cost-reduction.

\subsection{Current reference PV process and needs for cost-reduction}

In order to adopt an effective digital approach and support the cost reduction along the PV value chain, a first step is represented by the definition of a reference $\mathrm{PV}$ process map aimed to highlight the main work stages. This is useful to detect the current main barriers, bottlenecks and needs to reduce extra-costs, towards the definition of a digital PV process. This has been done thanks to the activities carried out within the SuperPV project and on the basis of literature review (e.g., [10-12]). A summary of the reference PV process and the main stages is shown in Figure 1.
The upstream process includes activities related to manufacturing of PV modules, inverters, O\&M equipment and other electrical devices, as well as racking and mounting structures. The downstream process encompasses the whole EPC (Engineering, Procurement, Construction) phase that incudes intensive information based activities such as the project design, permits and contracts, the project definition, the procurement, the construction and installation, and the O\&M.

From the literature review and from answers to the survey carried out within SuperPV Consurtium, it arises that the PV value-chain is characterized by a highly fragmentation. In particular, results of the survey show that there are some common informative bottlenecks among stages that can be the responsible of an inefficient and fragmented PV process. Most recurrent bottlenecks are described below:

- From manufacturing stage to project development

Lack of reliable information from manufacturers or distributors about components 'costs and about procurement and installation costs (which can also vary depending on the project location) for obtaining reliable assessment of the $\mathrm{PV}$ project cost in the project development stage.

- From project definition stage to manufacturers

Especially for the realization of customized products, missing of specific information for developing the customdesired product solution.

- From project definition stage to procurement Lack of interoperability/access to project definition information that may lead to mistakes to acquire the right products type and/or quantity for the PV project.

- From project definition stage to construction stage Lack of access to project definition information that may lead to mistakes in the assembly or installation failure. - From project definition stage to O\&M

Lack of detailed specifications about product data and system layout may lead to errors in the definition of the O\&M equipment and systems.

- O\&M - manufacturing

No access to historical data of performances of PV plant. These missing information, for instance, do not allow to improve products on the basis of real behaviour on the field and lessons or knowledge derived from past experiences. 


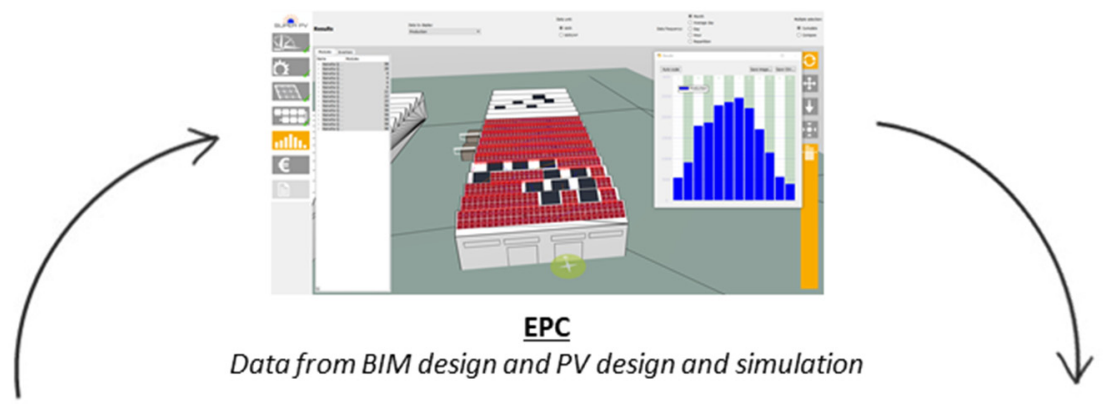

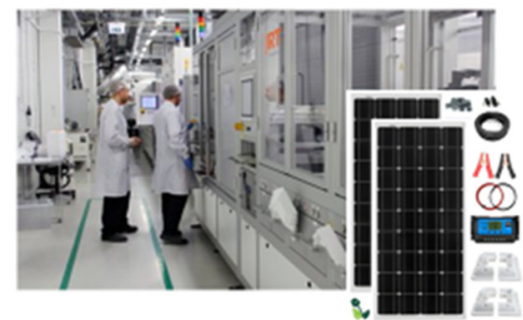

Manufacturing

Data of PV product and data from production lines

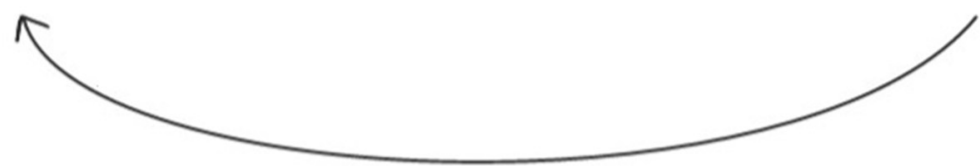

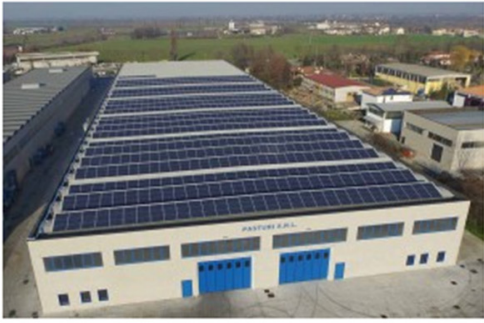

$\underline{0 \& M}$

Data from PV Asset management and maintenance

Fig. 2. Overview of the PIM approach.

The above mentioned bottlenecks highlight that data are the fundamental issue, mainly due to their lack from one stage to another or due to the non compatible format or level of detail. This involves inefficiencies and extra-costs along the PV process that weaken the competitiveness of the PV sector for medium and large PV plants. In order to try to avoid this fragmentation among the different workstages of the PV process, a more integrated and interoperable PV process should be established.

\subsection{Development of the PIM approach}

As it arises from the previous section, there is the need to improve information exchange among the different PV process stages, to support interoperability and to provide reliable data. In this context, the adoption of a digital, more open and collaborative approach based on data-sharing among different stakeholders and a more coordinated information workflow from the design till O\&M can be an enabler for the PV sector and play a key-role for optimizing the PV process and reducing costs.

In the last 20 years, the construction sector that typically is characterized by a high fragmentation and the involvement of several different stakeholders, adopted a new approach to overcome inefficiencies and reduce extracosts. This approach, named BIM (Building Information Management), has been demonstrated to be effective for the integration of different stakeholders' activities on building projects and for the efficiency of the process based on a collaborative digital environment that allows sharing data and information thus enhancing communication, reducing errors, saving time and optimizing costs $[13,14]$.

Considering the demonstrated advantages of the BIM approach in the construction sector, a similar approach can be envisioned and specifically tailored for the PV sector. In detail, a collaborative digital approach named PV Information Management (PIM) is introduced in this work for supporting an integrated and interoperable PV process aimed at cost-reduction and improvement of $\mathrm{PV}$ project's quality and performances. In a few words, the PIM approach can be defined as the framework for a digital $\mathrm{PV}$ process consisting of the main work stages and digital workflows to improve the efficiency of the current PV process and overcome current bottlenecks associated with cost and extra-costs along the value-chain.

Accordingly, the PIM approach tackles three main stages which are the manufacturing, the EPC and the O\&M. Specifically, the opportunity to share data of $\mathrm{PV}$ products from the manufacturing to the EPC (Engineering, Procurement, and Construction) stage allows transferring relevant data till the operation of the real PV plant. By monitoring these data on-site and analysing them, new informative patterns can arise not only to improve O\&M and reduce related costs but also to enhance other stages. For instance, this information can be used to provide manufacturers with feedback on PV product quality in a PLM (Product Lifecycle Management) perspective or to give feedback to the EPC stage about real performances of a specific project (Fig. 2). 
To really implement this approach, it is necessary to go beyond the creation of point solutions that provide punctual technologies for specific uses without creating a seamless workflow and changing how people work. For this reason, the SuperPV project introduces a prototype of a collaborative platform for design, simulation and operation of PV plants to reduce inefficiencies among the main key-stages and avoid related extra-costs.

\subsection{Economic assessment and cost analysis background}

In order to obtain seamless and integrated workflow along the $\mathrm{PV}$ process, it is necessary to adopt a wider perspective capable not only to consider technical aspects during but also to provide and upgrade related economic aspects since the preliminary design stage. By adopting the PIM approach, the engineering design stage is improved thanks to the integration of economic parameters and data directly associated to planned materials and components. In this way, the engineering design process is speeded up and combined techno-economic assessments - which are of fundamental importance for evaluating the investment, especially for medium and large PV plants- can be easily provided to the client. The main economic KPIs that are integrated within the PIM approach are the Capital Expenses (CAPEX) and the Levelized Cost Of Energy (LCOE).

\subsubsection{CAPEX}

Capital Expenditures, also CAPEX, are the initial total investment required to obtain an asset. In the case of medium and large PV systems, the asset can be represented by PV components (e.g., PV modules, power electronics, AC and DC materials, etc.), mounting structures, labour and installation costs, project indirect costs (e.g., engineering, procurement, construction management, etc.), owner costs, financial costs and permits. CAPEX can be also named $\mathrm{CC}$ and it is shown in equation (1).

$$
\begin{aligned}
C C= & C_{P V_{\text {mod }}}+C_{\text {inv }}+C_{D C / D C}+C_{\text {trafo }}+C_{\text {cable }} \\
& +C_{\text {comm }}+C_{\text {other }_{B} \text { oP }}
\end{aligned}
$$

where $C_{P V m o d}$ : cost of the PV modules; $C_{i n v}$ : cost of power inverters; $C_{D C / D C}$ : cost of DC/DC converters; $C_{\text {trafo }}$ : cost of transformer; $C_{\text {cable }}$ : cost of cables; $C_{\text {comm }}$ : cost of SCADA; $C_{\text {other_BoP: }}$ : cost of other components (e.g., mounting structures).

\subsubsection{LCOE}

The Levelized Cost Of Electricity (LCOE) is an economic comparative indicator that allows measuring the lifetime costs of generating electricity, thus helping the decisionmakers in finalizing a project. It is shown in equation (2) and defined as the discounted total lifetime cost (LCC) as shown in equation (3) divided by the discounted total lifetime energy generated by the PV system (NPE) as shown in equation (4).

$$
\begin{gathered}
L C O E(€ / M W h)=\frac{L C C}{N P E} \\
L C C(€)=I C+C C+\frac{D C}{(1+D R)^{t}} \\
+\sum_{i=1}^{t} \frac{C a p(i) L I+M(1+I)^{i}}{(1+D R)^{i}} \\
N P E(M W h)=\sum_{i=1}^{t} \frac{E(i)}{(1+D R)^{i}}
\end{gathered}
$$

where $t$ : lifetime in years; $I C$ : installation cost; $C C$ : the CAPEX; $D C$ : decommission cost; $D R$ : discount rate; $C a p(i)$ : capital cost of the year $i$; $L I$ : loan interest; $M$ : maintenance cost; $I$ : inflation rate; $E(i)$ : the energy produced during the year $i$.

\section{Prototype collaborative platform for design, simulation and operation}

The prototype collaborative platform for design, simulation and operation of PV system developed within the SuperPV project is designed in accordance with the PIM approach and it is envisioned as an interactive and interoperable platform enabling the different stakeholders (PV manufacturers, PV engineers, construction engineers, operations manager) to easily collaborate and share their PV data and information across the process stages and during the PV plant lifecycle.

Specifically, the adoption of the PIM approach within the software platform enables not only a cost reduction of PV systems through the design of competitive PV plants but also an indirect cost reduction thanks to the information management and sharing among the main stages of the downstream PV process, with a specifically focus on the EPC and O\&M stages.

The proposed software platform relies on the following pillars:

- The SuperPV software for design and simulation of large and medium $P V$ plants based on the existing software named BIMSolar ${ }^{\circledR}$ that allows simulating solar irradiation with hourly resolution thanks to a complex raytracing algorithm, designing BAPV and BIPV system, as well as simulating their energy performances.

- The SuperPV Digital Twin for the PLM of PV modules, which allows making the digital PV plant model (developed in the previous step) alive thanks to the connection with real data coming from the physical PV system. Specifically, this Digital Twin will be aimed at supporting the PLM (Product Lifecycle Management) perspective for PV modules.

The operative procedure is shown in Figure 3, and operative steps are the following: 


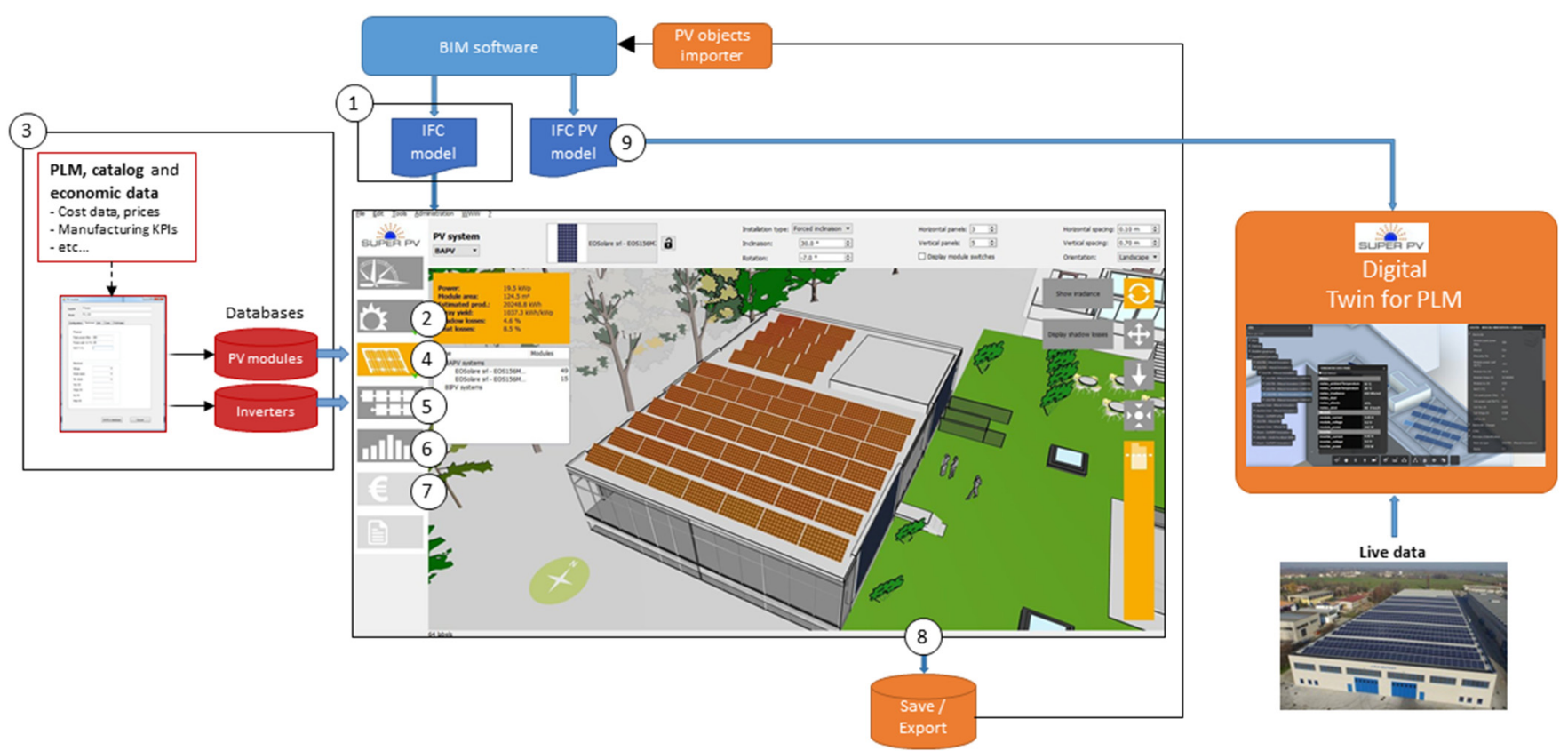

Fig. 3. SuperPV Platform: operative procedure.

- Input of an informative 3D digital model of context where the medium/large PV system will be implemented. Such a 3D model should include contextual objects that can affect the PV systems, such as nearby buildings, vegetation or mounting structures in order to be able to simulate the shadowing. Differently from other software for PV simulation, the SuperPV platform allows importing the digital model created through BIM software as IFC (Industry Foundation Classes) file. In the case of the SuperPV software, also models of terrains as IFC can be imported thus providing the opportunity to design and simulate ground-mounted PV plants. Indeed, BIM represents an approach more and more adopted not only in building sector but in the entire construction industry, involving civils and infrastructure works including energy. Moreover, since IFC is an open international standard (ISO 16739-1:2018), every digital model created in the BIM environment can be used. This promotes neutrality and opportunity to use a wide range of BIM software platforms.

- Calculation of local solar irradiance received on surfaces that allows the user identifying the most suitable surfaces on which to implement the PV system.

- Selection of the most appropriate PV modules from the database. The database of PV modules (as well as other database for inverters, glazing, etc.) is structured to include geometrical, technical, energy and cost specifications of each element. The database is filled automatically or manually with catalog data coming from the manufacturers or suppliers. It can also be loaded from external sources (e.g., PHOTON ${ }^{\circledR}$ database). In the SuperPV version, data and parameters associated to PV components are envisioned in a PLM (Product Lifecycle Management) perspective.
This means that data and properties of the components are stored within the file and are "transferred" from one stage to another in order to be accessible for updates or other purposes (e.g., maintenance) along the PV process.

- Design of the PV system geometrical layout and optimization of orientation, tilt angle and spacing to achieve the maximum energy yield, thanks to the decision-support tools described in Section 3.2.

- Definition of the PV system electrical layout (selection of inverters, wiring).

- Calculation of PV yield on annual, monthly and hourly basis.

- Cost assessment (e.g., material cost, total investment cost, O\&M costs, LCOE, etc.), through the functionalities described in Section 3.1.

- Export to BIM software (e.g., Autodesk Revit ${ }^{\circledR}$ ) for detailed drawings and execution plans.

- Import in the Digital Twin Platform, that is described in Section 3.3.

\subsection{Cost-management and economic estimation tool}

Beyond the evaluation of technical characteristics of PV system, also economic aspects are important indicators for $\mathrm{PV}$ investors. For this reason, it is fundamental to support the economic estimation of PV systems to have a better understanding of costs not only during the design stage but also during the operational life service. In order to achieve this twofold goal, two main calculation tools have been addressed in the SuperPV platform: the Capital Expenses (CAPEX) calculation tool and the Levelized Cost Of Energy (LCOE) calculation tool. 


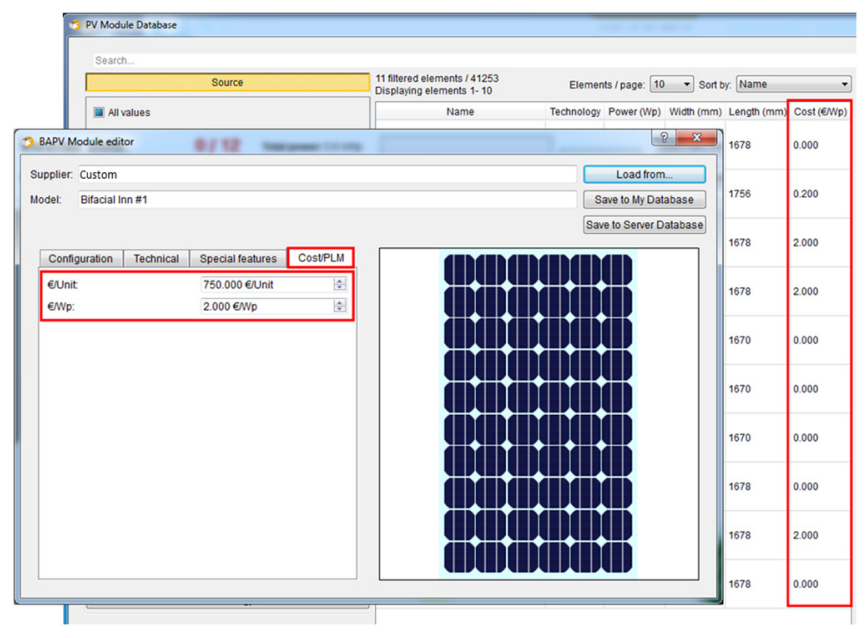

Fig. 4. Improved database of PV modules.

In order to provide the user with detailed CAPEX calculation, the database of PV modules and inverters has been enriched with parameters about costs (Fig. 4), whereas costs of cables are provided per unit of length. In this way, the investment costs for these components can be automatically calculated, and SCADA and other costs can be directly filled in by the user, thus obtaining the total investment cost.

These data are then integrated within the LCOE calculation tool, in accordance with formulas described in Section 2.3.2, to estimate the final LCOE value of the $\mathrm{PV}$ project. Also in this case, the tool provides the user a high flexibility since parameters such as discount rate, maintenance costs, inflation rate can be modified directly by the user to suit the economic analysis depending on the local context. As a result, the tool shows the main KPIs such as the final investment cost (CAPEX), the LCC, the NPE and the LCOE (Fig. 5).

\subsection{Decision support tools for PV engineers}

As it arises from the literature review and the survey, the design stage is characterized by several reworks and iterative tasks that involve extra-time and indirect extracosts. To avoid inefficiencies and support PV engineers in this stage, the tool for the optimization of the PV plant layout and the PV variants comparison tool have been introduced.

\subsubsection{PV plant layout optimization}

A decision support tool was developed for the optimization of the layout of $P V$ systems. It includes a functionality for maximizing the energy yield by an iterative mechanism that simulates the irradiation on different PV module' tilt, orientation and for different distances among PV modules. This mechanism searches through multiple steps for tilt, orientation and distances (variables), and finds the optimal ones that are needed to maximize the irradiation of the PV module surface (Fig. 6).

\subsubsection{Variants comparison tool}

To streamline the decision-making process is fundamental to take into account the most significant KPIs, not only in terms of energy aspects but also considering economic KPIs. Considering that the PV design process leads to develop several design variants and each of them has several techno-economic KPIs, a variants comparison tool has been introduced to enable the concurrent analysis of different parameters at a glance (Fig. 7). The main KPIs indeed are: installed power, AC production, CAPEX, Operational Costs (OPEX), Energy Yield and LCOE.

\subsection{Digital twin for PLM of PV modules}

The concept of the Digital Twin (DT) was introduced by Grieves and Vickers around 2003 as "a set of virtual information constructs that fully describes a potential or actual physical manufactured product from the micro atomic level to the macro geometrical level. At its optimum, any information that could be obtained from inspecting a physical manufactured product can be obtained from its Digital Twin" [15]. The DT origins in the field of Product Life-cycle Management (PLM) and, indeed, it is a concept that arises from the design stage with the digital and physical creation of the digital object that is installed in the physical environment. Once in place, the real object is monitored and managed during its operational stage and related information and data generated throughout its life-cycle are used to make the digital object alive as a Digital Twin. Indeed, the DT can be of two types: Digital Twin Prototype (DTP), that is created in the design stage of a product and/or asset, and Digital Twin Instance (DTI), that starts life as when the product/asset is realized. The aggregation of multiple DTIs (e.g., due to several product/assets) forms the Digital Twin Aggregate (DTA). All these different types of DT exist "within the Digital Twin Environment - the virtual representation of the environment within which the physical product exists - that enables virtual techniques such as simulation, modelling, and evaluation" [16]. In this way, a PLM approach can be enabled: product/assets information can be collected along the whole lifecycle and stored in a virtual environment, where analyses can be also carried out to increase knowledge of current products, as well as to improve future new products.

In the SuperPV project, the software for PV design and simulation allows obtaining a digital model of the PV plant with embedded information. This model can be considered as Digital Twin Prototype (DTP), namely the prototype of the physical PV asset including information about physical attributes, properties, operating parameters, costs of materials, etc. When the Digital Twin Prototype is validated with as-built examination, the validated DTP can be directly used for the O\&M. Indeed, after the realization of the PV plant and thanks to the collection of IoT data from the physical site, the DTP can be imported in the Digital Twin Platform where the "static" digital model of PV modules become Digital Twin Instances (DTI). The DTI can be considered an "evolution" of the DTP since it is a digital "live" representation of an object 


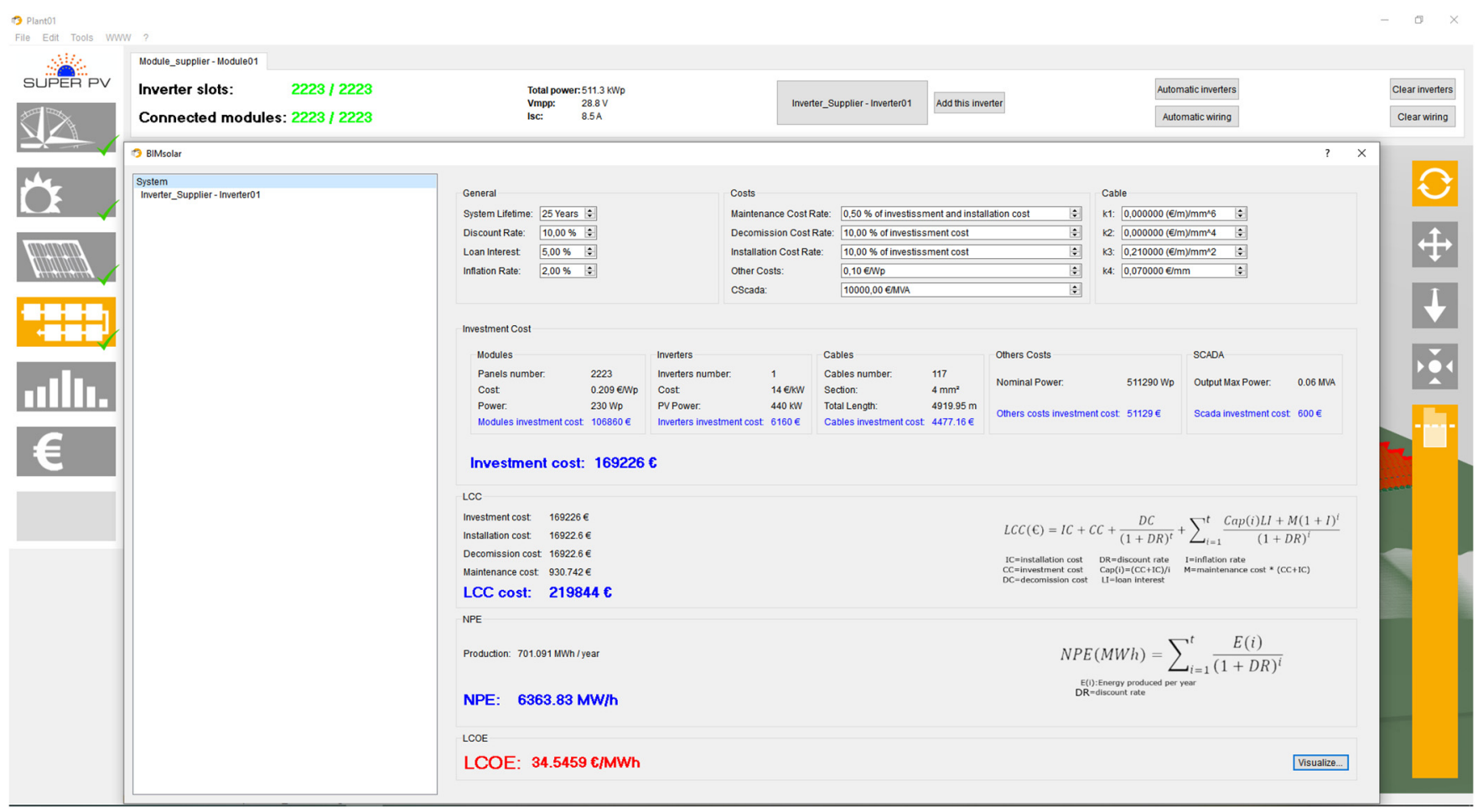

Fig. 5. Graphical interface for the calculation of economic KPIs.

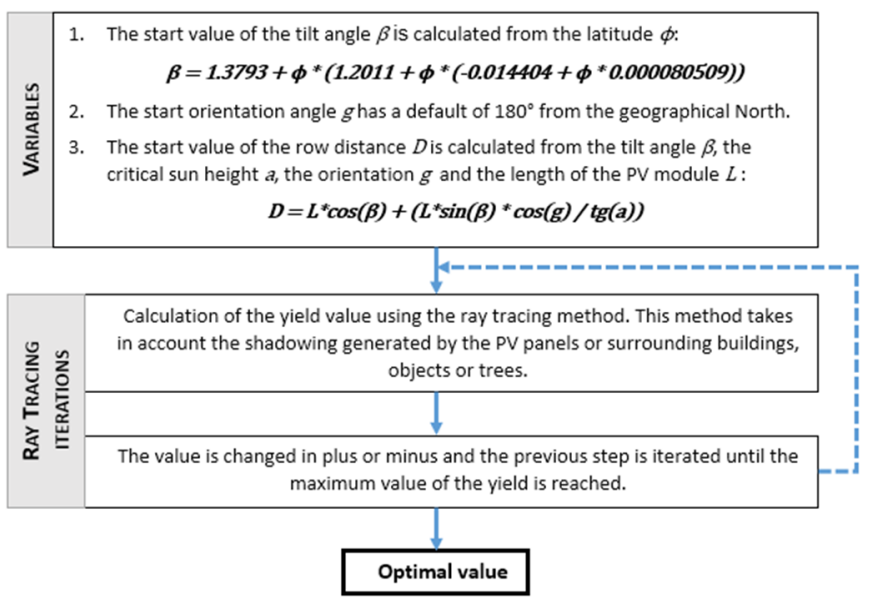

Fig. 6. Optimization workflow for PV plant layout.

that "remain linked throughout the life of the physical product" [16] thanks to specific communication devices and/or IoT sensors. The aggregation of these DTIs creates what is called a Digital Twin Aggregate (DTA) that during the operation of the $\mathrm{PV}$ plant allows the real-time monitoring and the possibility to record the whole lifecycle of PV modules. Through this workflow, the Digital Twin Environment (DTE) is obtained, as shown in Figure 8.

In accordance with the PIM approach, the SuperPV Digital Twin for the PLM of PV systems represents a strategical solution to monitor real performances of $\mathrm{PV}$ modules thanks to real-time monitoring sensors, store PV module data and analyse them along the life-cycle. The
PLM of PV modules through Digital Twin can play an important role to achieve sustainability goals in a wider perspective. Indeed, considering the transition towards a circular economy approach, it is more and more important to ground on reliable information about the whole product life-cycle and reliable data about its materials and resources in order to effectively analyse remaining value and performances so that a second operational life could be supported minimizing waste generation. At the present moment, the DT for PLM of PV modules in underdevelopment will be provided as a technological framework where PV specialists can develop models to estimate residual life-time of $\mathrm{PV}$ modules thanks to collected information.

\section{Results and discussion}

\subsection{Preliminary validation on a real case study}

In order to demonstrate the reliability of the proposed software for design and simulation, a real case study has been used to benchmark the results of the SuperPV software with real data and PVSyst [17] results. The reference PV system is a standard BAPV system located in Genève (Switzerland) with a peak power of $3.6 \mathrm{~kW}$, southeast orientation ( $-67^{\circ}$ azimuth) and $27^{\circ}$ tilt (Fig. 9).

A 1-year data with monthly resolution has been collected thanks to the inverter monitoring system. Moreover, real raw climatological data have been obtained from the Swiss meteorological database thanks to the IdaWeb service of the Swiss Federal Office of Meteorology and Climatology (MeteoSwiss) [18]. The nearest weather 


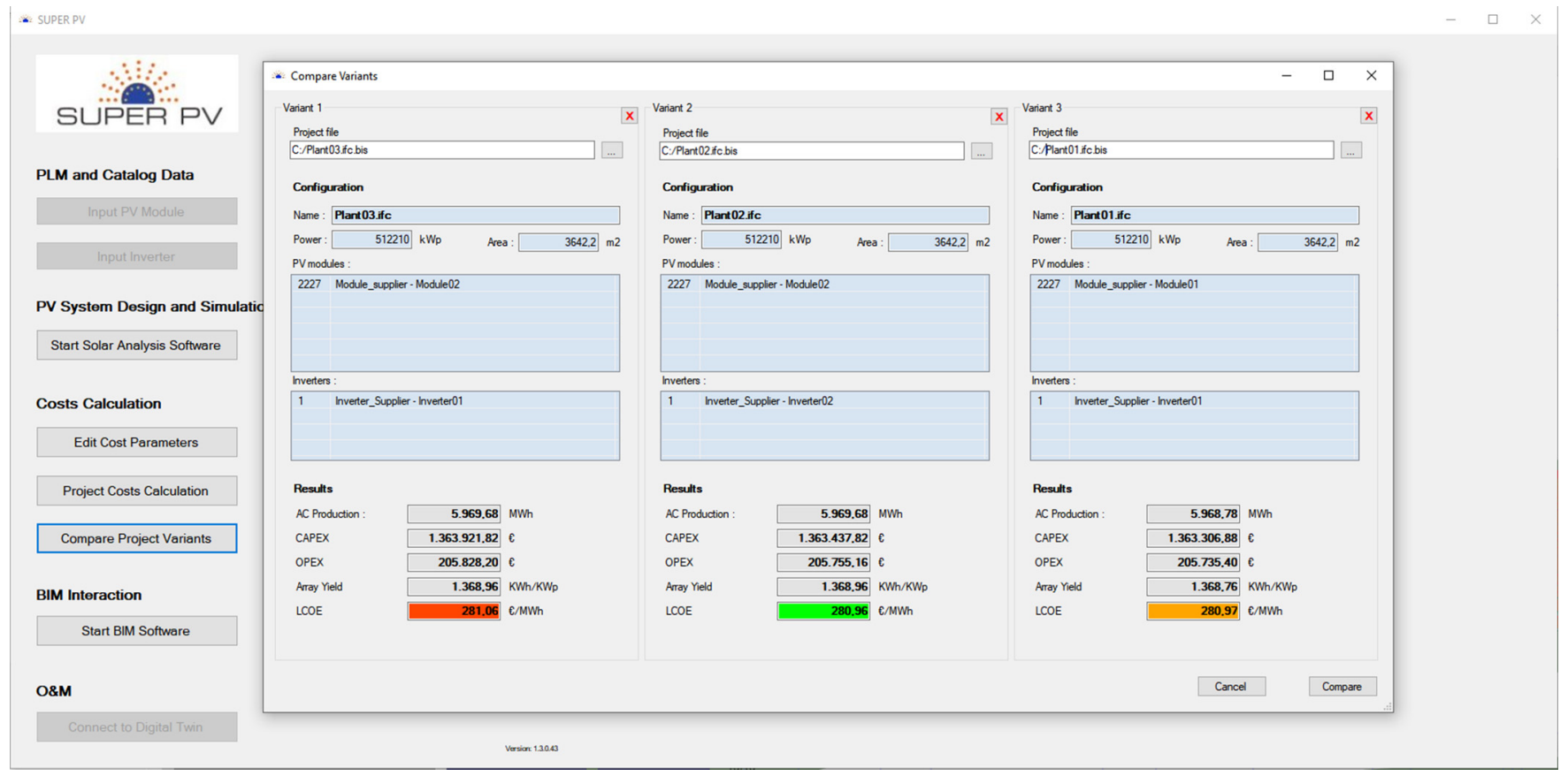

Fig. 7. Graphical interface for the PV variants comparison.

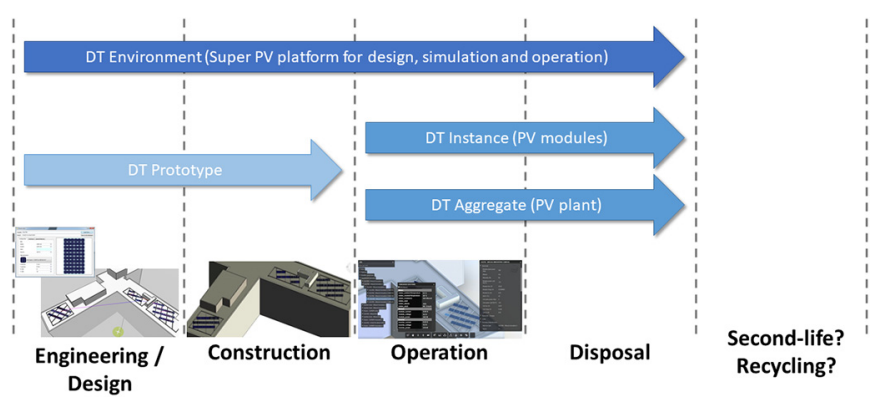

Fig. 8. Digital Twin along the PV process.

station has been identified in the Prairie area in Genève (about $4 \mathrm{~km}$ far from the case study) and it has been used as reference to collect real meteorological data with hourly resolution. Data about irradiation (GHI, DHI, DNI), air temperature, dew point temperature, relative humidity, air pressure, wind direction and wind speed have been used to create the input weather file. The local weather file has been used for both SuperPV and PVSyst simulations. In both simulations, the real PV system has been modelled with the real PV components (PV modules, inverter, cablings, etc).

Comparison between SuperPV and PVsyst in terms of simulated irradiation and the real energy output are shown in Figure 10. Specifically, differences in terms of irradiation between SuperPV and PVSyst are in the range 0\% and 6\%, with a mode value of $2 \%$ and an average value of $3 \%$ with maximum differences that occur during November and December.

Figure 11 shows the values of monthly production collected from the inverter monitoring system, and from the SuperPV and PVSyst simulation results. Regarding energy production, differences among SuperPV and PVSyst are in the range $1 \%$ and $6 \%$, with a mode value of $4 \%$ and an average value of $3 \%$ with a maximum difference that occurs in December.

It is important to highlight that both software overestimate the $\mathrm{PV}$ production but this is probably due to the input weather file that includes data about a location situated $4 \mathrm{~km}$ away from the real site. From this preliminary assessment, it arises that small differences appear between SuperPV and PVSyst. Considering that the input weather file is the same, this can be due to the different models for estimating PV production. However, a larger datasets and larger PV systems should be analysed to obtain a significant validation of the SuperPV software.

\subsection{Preliminary cost-reduction assessment}

PV cost-reduction represents the overall goal of the SuperPV project. Among strategies and innovations developed to achieve this goal, one is represented by the adoption of the PIM approach and the consequent introduction of the collaborative platform for design, simulation and operation of PV system. Indeed, such a digital platform has the goal to support the global optimization of PV systems by including not only technical parameters estimation but also the economic KPIs assessment, namely the CAPEX and LCOE. By integrating techno-economic KPI, the cost of PV can be reduced in the design stage. Beyond that, this platform addresses collaborative approach in the PV sector through a seamless workflow that allows transferring data from the design stage to the O\&M stage. Also in this way an indirect cost-reduction in the PV process can be achieved. 


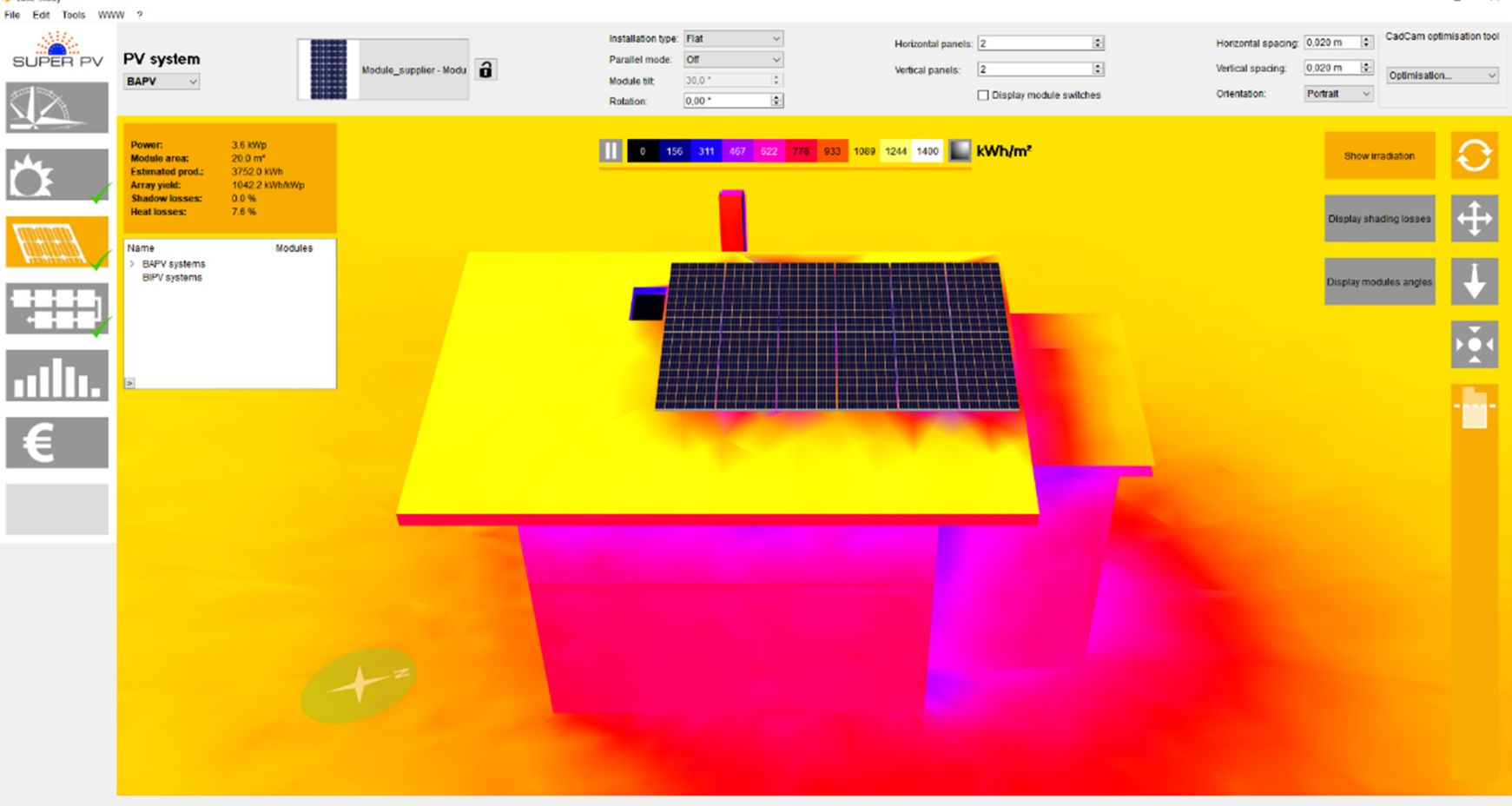

Fig. 9. PV case study in the SuperPV software for design and simulation.

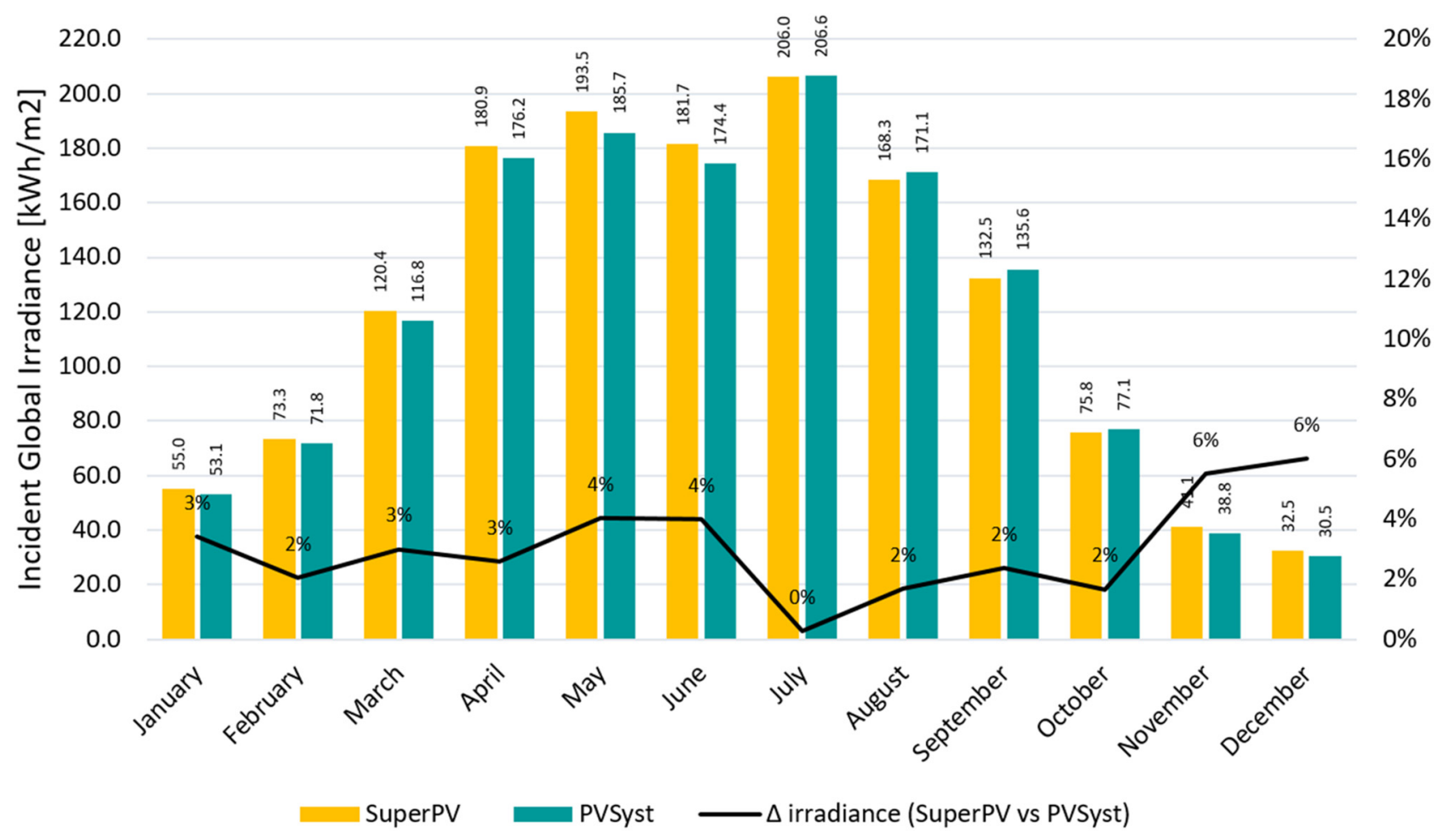

Fig. 10. Incident Global Irradiance on PV modules. 


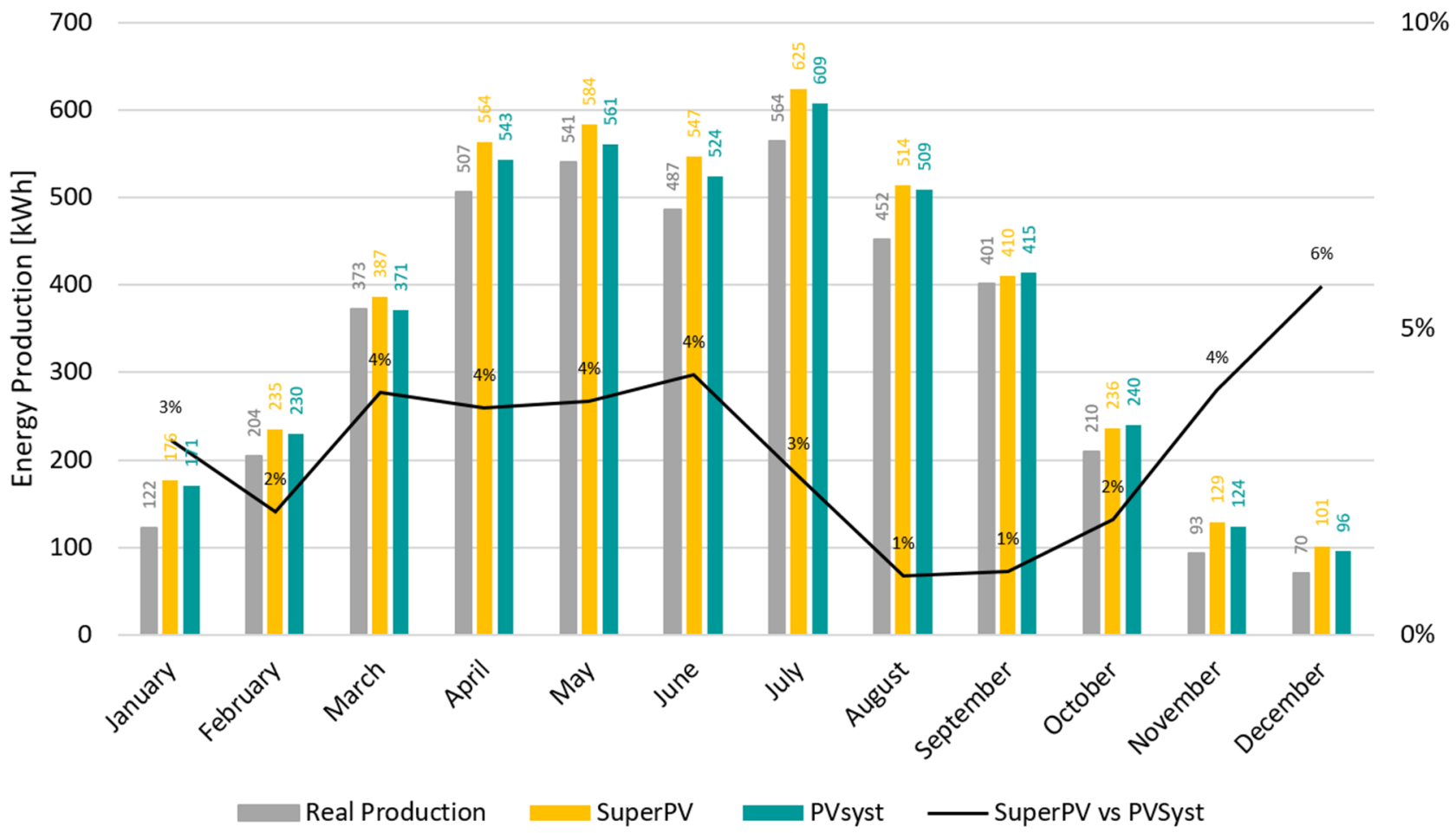

Fig. 11. Comparison among real PV production, SuperPV results, PVSyst results.

Considering the prototype stage of the platform, only a preliminary assessment of such an indirect impact of the proposed solution on the cost reduction can be carried out. The preliminary assessment has been performed through a short survey to a small group of PV stakeholders with experience in PV plant engineering and design (2 PV manufactures and $1 \mathrm{PV}$ engineer) that has been involved in an online training session on the SuperPV platform. Participants have been asked to provide feedback about the potential impact (less than 10\%, 10-20\%, 20-30\%) of the proposed innovations to the cost-reduction in the different stages of the EPC and O\&M stages of the PV process, as shown in Table 1.

Even though the SuperPV platform is still a prototype under development and the survey should be extended to a larger number of PV stakeholder, preliminary results obtained by considering the most frequent answers show that the whole impact on cost-reduction due to the proposed innovations is in the range between $14 \%$ (lower value) and $55 \%$ (higher value). In particular, the feature that affect the most the cost-reduction is represented by the seamless workflow to obtain a Digital Twin for the O\&M phase of the PV plant. However, if also the impact on client engagement and understanding is considered, the impact of the platform rises in the range of $25 \%-74 \%$. In a second stage, a larger reference group of $\mathrm{PV}$ stakeholders will be involved in the cost reduction assessment of the full-developed version of the platform, and thanks to other activities involved in the SuperPV project additional cost savings related to failures detection and preventive maintenance of PV modules will be estimated.

\section{Conclusions}

The main goal of this paper was to propose a novel PIM approach to support the cost reduction along the work-stages of the PV process, thanks to the benefits and advantages provided by digitalization. Indeed, there is a strong need of improving the current $\mathrm{PV}$ value-chain and enhancing the information exchange among the different stakeholders and work-stages, not only for reducing risks and unforeseen extra-cost, but also for improving the PV project quality, reliability and performances.

Therefore, as technical result of the PIM approach, a prototype collaborative platform for design, simulation and operation of medium and large PV systems has been implemented within the SuperPV project. This platform allows the user to embed both technical and economic aspects to drive the design process in a seamless workflow providing the main KPIs needed to evaluate the investment for different design scenarios of a PV plant. Moreover, the platform allows to easily transfer the "as-built" digital model of the PV plant (and related information and parameters) to the O\&M stage where the PV plant can be made alive with real data coming from PV components and sensors, thus obtaining a Digital Twin. The potential 
Table 1. Potential cost reduction impact of SuperPV platform depending on the engineering design step and O\&M.

\begin{tabular}{|c|c|c|c|c|c|c|}
\hline \multirow{2}{*}{$\begin{array}{l}\text { Impacts due to the proposed } \\
\text { new functionalities introduced } \\
\text { in the SuperPV platform }\end{array}$} & \multicolumn{2}{|c|}{ Mode Value } & \multicolumn{2}{|c|}{ Mean Value } & \multicolumn{2}{|c|}{ Total Impact } \\
\hline & $\begin{array}{l}\text { Lower } \\
\text { Value }\end{array}$ & $\begin{array}{l}\text { Upper } \\
\text { Value }\end{array}$ & $\begin{array}{l}\text { Lower } \\
\text { Value }\end{array}$ & $\begin{array}{l}\text { Upper } \\
\text { Value }\end{array}$ & $\begin{array}{l}\text { Lower } \\
\text { Value }\end{array}$ & $\begin{array}{l}\text { Upper } \\
\text { Value }\end{array}$ \\
\hline $\begin{array}{l}\text { Impact on Design (e.g., } \\
\text { import of 3D CAD/BIM } \\
\text { model of the context, PV } \\
\text { module creation, geometrical } \\
\text { design of the PV plant) }\end{array}$ & $1 \%$ & $9 \%$ & & & & \\
\hline $\begin{array}{l}\text { Impact on energy yield } \\
\text { estimation (e.g., electrical } \\
\text { design and simulation) }\end{array}$ & $1 \%$ & $9 \%$ & & & & \\
\hline $\begin{array}{l}\text { Impact on economic } \\
\text { estimation and cost-control } \\
\text { (e.g., CAPEX and LCOE } \\
\text { calculation) }\end{array}$ & $1 \%$ & $9 \%$ & & & $14 \%$ & $55 \%$ \\
\hline $\begin{array}{l}\text { Impact on rework and } \\
\text { iterative tasks (e.g., ease to } \\
\text { change PV components, } \\
\text { variants comparison) }\end{array}$ & $1 \%$ & $9 \%$ & & & & \\
\hline $\begin{array}{l}\text { Impact on O\&M (seamless } \\
\text { workflow) }\end{array}$ & & & $10 \%$ & $19 \%$ & & \\
\hline
\end{tabular}

impact of the proposed platform in terms of cost reduction in the $\mathrm{PV}$ process is preliminarily estimated in the range from $14 \%$ (conservative approach) to $55 \%$ (maximum potential approach).

Future developments of this activity within the SuperPV project are of different natures. On one hand, a more detailed cost reduction assessment will be carried out introducing new functionalities required by real PV stakeholders and improving current tools grounding on feedback from real PV players. On the other hand, the Digital Twin envisioned for the PLM approach of PV modules will be further developed to obtain a first prototype on a real case study.

This paper has been developed in the framework of the SuperPV project. The SuperPV project has received funding from the European Union's Horizon 2020 Research and Innovation Programme under Grant Agreement No. 792245. Authors would like to thank also IREC (specifically, Dr. J. L. Dominguez and Mr. P. Paradell Solà) for the support in the economic assessment, as well as BNW-ENERGY (Dr. T. Haarberg) and Becquerel Institute (specifically, Mr. P. Macè and Ms. E. Bosch) for their supervision in economic assessment.

\section{Author contribution statement}

Conceptualization: S.E. and B.P., Writing: S.E., B.P. and M.W., Software development and prototyping: M.W. and N.V.K, Supervision and revision: F.F. and N.V.K.

\section{Appendix A: Nomenclature}

\begin{tabular}{ll}
\hline BIPV & Building-Integrated Photovoltaics \\
\hline CAPEX & Capital Expenses \\
DC & Direct Current \\
DHI & Diffuse Horizontal Irradiance \\
DNI & Direct Normal Irradiance \\
DT & Digital Twin \\
DTA & Digital Twin Aggregate \\
DTE & Digital Twin Environment \\
DTI & Digital Twin Instance \\
DTP & Digital Twin Prototype \\
EPC & Engineering, Procurement and Construction \\
GHI & Global Horizontal Irradiance \\
IFC & Industry Foundation Classes \\
LCC & Total lifetime cost \\
LCOE & Levelized Cost of Electricity \\
NPE & Total lifetime energy generated \\
OPEX & Operational Costs \\
O\&M & Operation and Maintenance \\
PIM & PV Information Management \\
PLM & Product Lifecycle Management \\
SCADA & Supervisory Control And Data Acquisition \\
\hline
\end{tabular}




\section{References}

1. Reuters Event, A European Glimpse into a Digitalised Solar PV. White Paper (2021)

2. L. Brett, The digitalisation of PV operations, Energize online magazine Nov./Dec. 2019, 20 (2019), https://www.ener gize.co.za/features/energize-novdec-2019/view-pdf

3. International Renewable Energy Agency, Innovation landscape brief: Internet of Things. Report (2019)

4. S. Alsadi, T. Khatib, Photovoltaic power systems optimization research status: a review of criteria, constrains, models, techniques, and software tools, Appl. Sci. 8, 1761 (2018)

5. N. Gui, J. Li, Y. Dong, Z. Qiu, Q. Jia, W. Gui, G. Deconinck, BIM-based PV system optimization and deployment, Energy Build. 150, 13 (2017)

6. M. Lovati, J. Adami, D. Moser, Open source tool for a better design of BIPV + battery system: an applied example, in $E U$ PVSEC Conference Procedings (2018)

7. A. Booth, N. Patel, M. Smith, Digital transformation in energy: Achieving escape velocity (2020), https://www. mckinsey.com/industries/oil-and-gas/our-insights/digitaltransformation-in-energy-achieving-escape-velocity

8. BIMSolar ${ }^{\circledR}$ software. https://www.bim-solar.com/en/bim solar-4/

9. SuperPV project. https://www.superpv.eu/

10. H. Ossenbrink, A.J. Waldau, N. Taylor, I.P. Pascua, S. Szabó, Perspectives on future large-scale manufacturing of PV in Europe, JRC Report No JRC 94724 (2015)

11. International Finance Corporation - World Bank Group. Utility-Scale Solar Photovoltaic Power Plants: A project developer's guide (2015), https://www.ifc.org/wps/wcm/ connect/a1b3dbd3-983e-4ee3-a67b-cdc29ef900cb/IFC+Solar + Report_Web + _08+05.pdf?MOD $=$ AJPERES\&CVID $=$ kZePDPG

12. C. Tjengdrawira, D. Moser, U. Jahn, M.V. Armansperg, I.T. Theologitis, M. Heisz, PV Investment Technical Risk Management: Best Practice Guidelines for Risk Identification, Assessment and Mitigation. Solar Bankability Project Report (2017), https://www.tuv.com/content-me dia-files/master-content/services/products/p06-solar/solardownloadpage/solar bankability d 5.8 best-practiceguidelines-for-risk-mānagment.pdf

13. B. Daniotti, A. Pavan, S.L. Spagnolo, V. Caffi, D. Pasini, C. Mirarchi, Benefits and challenges of BIM in construction, in BIM-Based Collaborative Building Process Management (Springer, 2020)

14. L. Doumbouya, G. Gao, C. Guan, Adoption of the building information modeling (BIM) for construction project effectiveness: the review of BIM benefits, Am. J. Civil Eng. Architect. 4, 74 (2016)

15. M. Grieves, J. Vickers, Digital twin: mitigating unpredictable, undesirable emergent behavior in complex systems, in Transdisciplinary Perspectives on Complex Systems (Springer, 2017)

16. D. Jones, C. Snider, A. Nassehi, J. Yon, B. Hicks, Characterising the digital twin: a systematic literature review, CIRP J. Manufactur. Sci Technol. 29, 36 (2020)

17. PVSyst ${ }^{\circledR}$ software. https://www.pvsyst.com/

18. IDAWEB. https://www.meteosvizzera.admin.ch/home/ser vizi-e-pubblicazioni/consulenza-e-servizi/portale-dati-per-linsegnamento-e-la-ricerca-idaweb.html

Cite this article as: Erika Saretta, Pierluigi Bonomo, Willy Maeder, Van Khai Nguyen, Francesco Frontini, Digitalization as a driver for supporting PV deployment and cost reduction, EPJ Photovoltaics 13, 1 (2022) 